\title{
Fuzzy based methodologies comparison for high-impedance fault diagnosis in radial distribution feeders
}

ISSN 1751-8687

Received on 5th September 2016

Revised 25th November 2016

Accepted on 17th January 2017

E-First on 27th February 2017

doi: 10.1049/iet-gtd.2016.1409

www.ietdl.org

\author{
Mauro S. Tonelli-Neto ${ }^{1}$, José Guilherme M.S. Decanini², Anna Diva P. Lotufo ${ }^{3}$, Carlos Roberto Minussi ${ }^{3} \bowtie$ \\ ${ }^{1}$ Instituto Federal de Educação, Ciência e Tecnologia de São Paulo (IFSP), Câmpus Votuporanga, Avenida Jerônimo Figueira da Costa, 3014, \\ 15503-110, Votuporanga, SP, Brazil \\ ${ }^{2}$ Instituto Federal de Educação, Ciência e Tecnologia de São Paulo (IFSP), Câmpus Presidente Epitácio, Rua José Ramos Júnior, 27-50,
} 19470-000, Presidente Epitácio, SP, Brazil

${ }^{3}$ Electrical Engineering Department, Campus of Ilha Solteira, UNESP, Universidade Estadual Paulista, Av. Brasil 56, P.O. Box 31, 15385-000 Ilha Solteira, SP, Brazil

凶E-mail: minussi@dee.feis.unesp.br

\begin{abstract}
This study presents a comparison of two developed intelligent systems that carries out, in an integrated manner, failure diagnosis on electric power distribution feeders. These procedures aim to identify and classify critical situations, as highimpedance faults, which can potentially damage the system components and cause power supply interruptions to consumers. The intelligent systems combine the wavelet transform, Dempster-Shafer evidence theory, voting scheme, fuzzy inference system and artificial neural networks. Results show the efficiency, reliability, and robustness of the proposed methodology, allowing its real-time application.
\end{abstract}

\section{Introduction}

Expansions in the electrical system, due to urban growth, have led to an increase in investments applied to developing new techniques/technologies and protection philosophy. These investments aim to provide automation to electrical distribution systems; i.e. their devices and operations [1, 2]. Devices automation is related to the use of artificial intelligence concepts, enabling the device to detect, classify, locate, and eliminate abnormalities without operator intervention [3]. The automation of operations, which is a result of the devices automation, intends to render the system proactive; i.e. the maintenance process becomes more direct and efficient due to the anticipation of information related to failures identified on the feeder [2]. Thus, the operation centre, based on the additional information, can inform the maintenance group in advance, reducing the time necessary to restore the normal operating state, which thus increases the reliability and power quality indices $[1,4,5]$.

In its current state, the distribution system has low monitoring and reliability levels for the services available to consumers. The low monitoring level is related to the information acquired from the feeder; e.g. voltage and current, which are, in most cases, measured only at the main substation. On the other hand, the low reliability is due to the diagnosis methods, which are based on the visual inspection of oscillographs by the operators and, therefore, are strongly dependent on their analysing experience. When the management centre relies only on human operators, the process is liable to errors, which, in an extreme case, can cause power supply interruption. Thus, without detailed and complete information about each part of the feeder, the role of the operator in determining possible failure events becomes even more complex, leading to losses for the utilities and consumers [6].

The electric power distribution system, in comparison with the transmission system, presents a predominantly radial topology with several branches, which makes it more susceptible to failures. These failures can be caused by animals touching energised parts of the network, wildfire, lightning, or human action; e.g. vandalism and depredation. In several cases, failures are easily detected and located. For example, short circuits on the feeder are identified in a reactive way; i.e. consumers who observe an abnormality in the energy supply communicate this event to the utility. Then, after mapping of the telephone calls, a maintenance group is sent to the probable failure area to restore the feeder operation to its normal state. However, there are failures that cannot be identified by the protection systems due to their singular behaviour, e.g. highimpedance faults (HIFs). Usually, this kind of failure is classified as normal operation; i.e. a feeder operating with an HIF does not show any evidence of defect or interruption in the energy supply. An HIF occurs when an energised conductor, whether broken or not, comes into contact with high-impedance surfaces (e.g., asphalt, sidewalk, and sand) or high-impedance objects (e.g., tree branches). The HIF limits the fault current to values below those detected by the protection devices, persisting on the feeder for hours, days, or weeks, which causes high power drain in the distribution system. Moreover, the current and voltage signals of the HIF have a random behaviour due to non-linearity and the possible presence of an electrical arc. Studies have described the distribution feeder operating in the presence of an unobserved/ undetected HIF, which is identified only when it has evolved into a critical failure, damaging many devices connected to the electrical network and interrupting the energy supply [7-9].

Thus, considering the technical and philosophical restructuring of the distribution system, utilities must aim for the development and implementation of methodologies to identify HIFs when other kinds of failures; e.g. short circuits and voltage disturbances, have been ruled out as the principal cause of problems in the energy supply. To this end, studies have been performed combining signal processing techniques, intelligent systems, and data fusion concepts. Below, the main proposals available in the specialised literature are highlighted.

A model description for representing HIFs in electrical distribution systems is presented in [10]. The model is based on a non-linear resistance representing the high impedance path during the critical event. An algorithm for HIF detection in electrical distribution systems evaluated taking into account several electric variables associated to HIFs. Field measurement and computer simulations validate the HIF model and the proposed detection algorithm.

A new HIF detection method using time-frequency analysis for feature extraction is proposed in [11]. A pattern classifier is trained, which feature set consists of current waveform energy and normalised joint time-frequency moments. The proposed method 


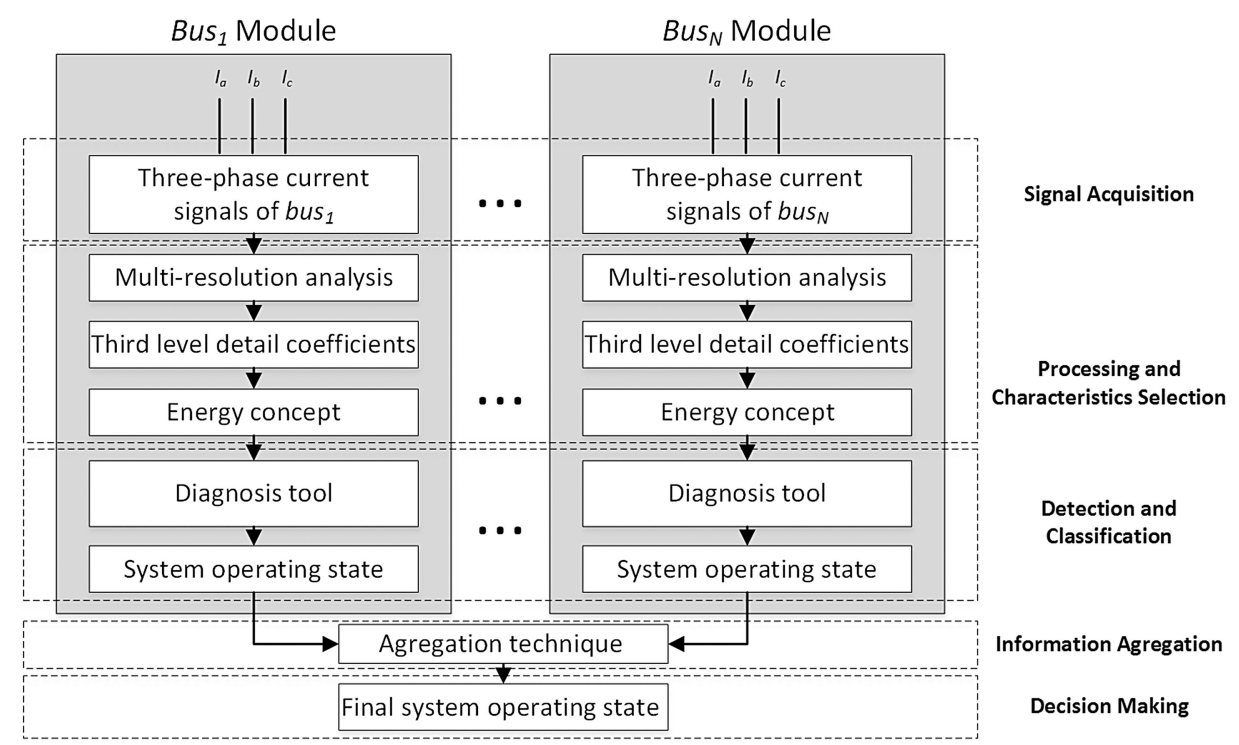

Fig. 1 Structure of the HIF diagnosis modules

shows high efficacy in all of the detection criteria. The performance was verified using real-world data, acquired from HIF tests on three different materials (concrete, grass, and tree branch) and under two different conditions (wet and dry).

Macedo et al. in [12] present a new methodology for HIFs detection in distribution systems based on interharmonic current signatures. The functionality of the proposed methodology was carried out with field tests on different types of soil. Results show great promise when it comes to detecting this disturbance.

The main aim of Batista et al. in [13] was to present a method for analysis of power systems transient events. The orthogonal component decomposition technique was tested aided by a real fault oscillogram recorded at a power distribution substation. Computer simulations showed that decomposition by orthogonal components was able to extract great qualitative information of fault occurrence and location with immunity to fault resistance effect.

A real-time methodology development is discussed in [14]. Using the energy of wavelet coefficients with border distortions, the HIFs are detected by its generated transients. The performance of the proposed wavelet-based method was assessed with compact and long mother wavelets by using data from staged HIFs on an actual energised power system, taking into account different fault surfaces, as well as simulated HIFs. Results show that the proposed method is reliable and accurate than other evaluated wavelet-based algorithms.

From this perspective, this work presents two methodologies for the diagnosis of HIFs in electric power distribution systems, combining three key concepts: signal processing techniques, intelligent systems, and sensor/data fusion. The employment of the wavelet transform focuses on the time-frequency domain signatures extraction, where peculiar transients generated by HIF are easily observed. Therewith, the two presented methodologies become robust and reliable even when occur minimal (unidentified) variations in the distribution feeder caused by an HIF. Besides that, the use of intelligent systems aims to automate the diagnosis process. Fuzzy based methods were chosen due to the following characteristics when applied to failure detection and classification: efficiency, robustness, reliability, and low computational effort, highlighting the possibility to incorporate new patterns, i.e. inclusion new information different from those previously acquired without needing to reinitialise the training step. Furthermore, the small number of indices used as input in the diagnosis procedure reduces the implementation costs and simplify its application in real time. Lastly, the theory of evidence aggregates the final results making the methodologies immune to human errors in decision-making process.

The first diagnosis methodology uses current oscillographies from different busses of the feeder. Multiresolution analysis and the energy concept are combined to extract the signals characteristics.
Therefore, signal representative indices compose the input vector of multiple fuzzy inference systems (FISs), which carry out a diagnosis of the system operating state. This multiple analysis provides greater robustness to the diagnosis process. Then, the evidence theory is used to aggregate all information produced by each FIS, providing probabilities referring to the possible system operating state, i.e. normal operation or HIF presence.

In the second methodology, the signal characteristic index is employed as input of multiple fuzzy-ARTMAP neural network (FANN), which provides the system operating state. The final probability of each of the operating states is generated by a voting process.

Then, the main objective of this paper is to provide a detailed evaluation considering the advantages and the disadvantages of the diagnosis techniques, which employs fuzzy concepts, and its influence in the final result, taking into account the principal attributes for the distribution system automation process: efficiency, reliability, processing time, possibility of including new patterns, among others.

\section{HIF diagnosis}

Alternatives methodologies have been developed to reduce the operator's dependence on the HIF identification process. Besides carrying out the detection, these methodologies indicates in which phase the HIF occurred, e.g. phases $a, b$, or $c$, aiding the posterior elimination by the maintenance group. In this paper, the methodologies use signal processing techniques, however the first uses the FIS and the Dempster-Shafer evidence theory to detect and classify HIF, whilst the second uses FANN and a voting scheme. Fig. 1 presents the procedures flowchart.

\subsection{Multi-resolution analysis and the energy concept}

One of the most important steps in the failure detection and classification process is the extraction of characteristics. Due to the HIFs present random behaviour and low fault current, several signal processing techniques have been employed to allow its diagnosis. These techniques must be able to highlight singularities to distinguish normal operation of HIFs. To illustrate the affirmative above, Fig. 2 shows the current oscillographies of system's normal operation and an HIF.

As can be observed, if the operator executes a visual inspection in these oscillographies, a correct diagnosis is not possible to be provided. Thus, applying signal processing techniques is fundamental for a correct diagnosis. The Fourier transform [15] and the wavelet transform $[16,17]$ are among the most common techniques used to signal analysis encountered in the specialised literature. Although the Fourier transform is widely used in signal processing, it has limitations that prevent its use in modern 

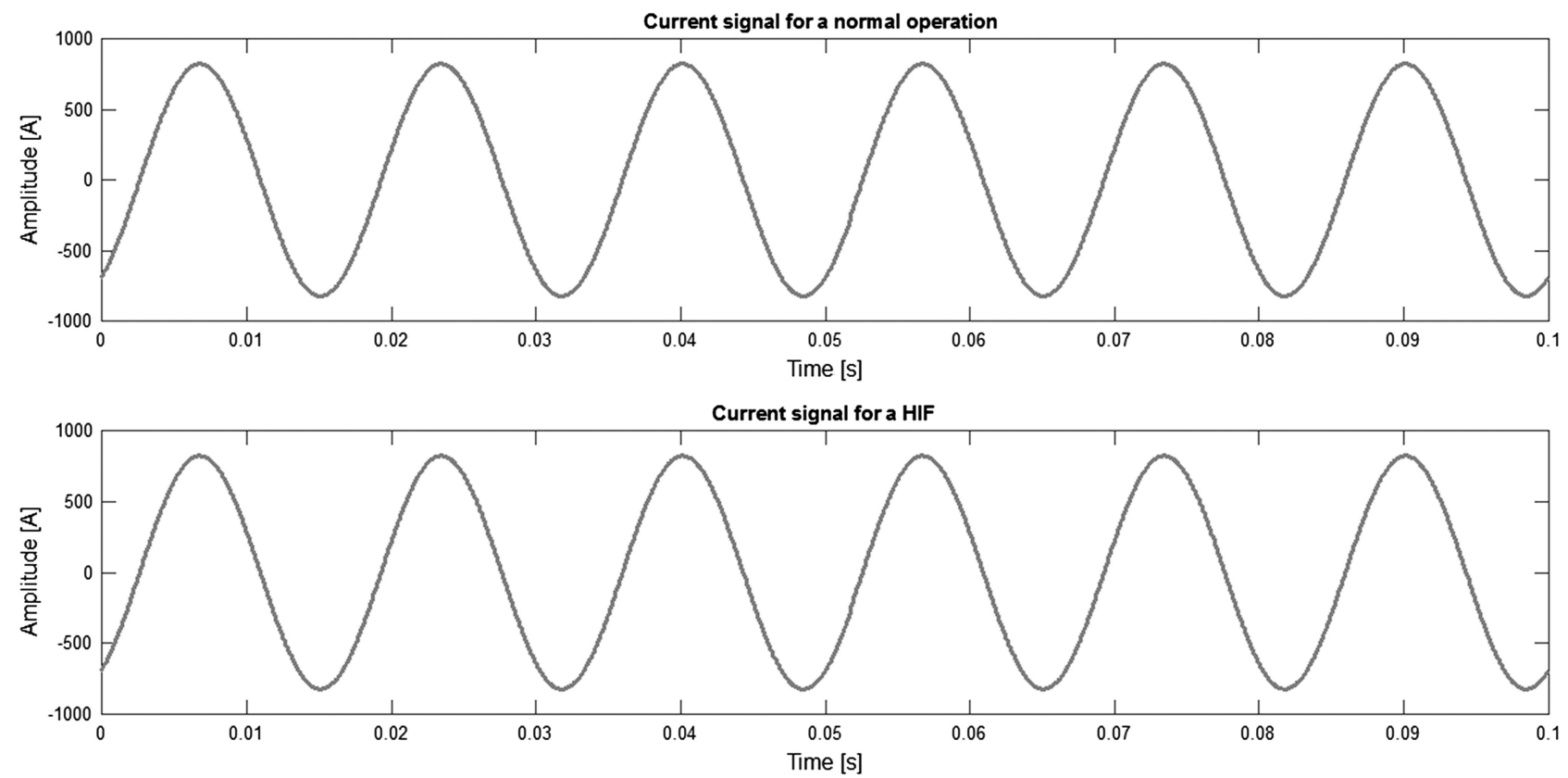

Fig. 2 Current oscillographies of system's normal operation and an HIF

Detail coefficients for a normal operation
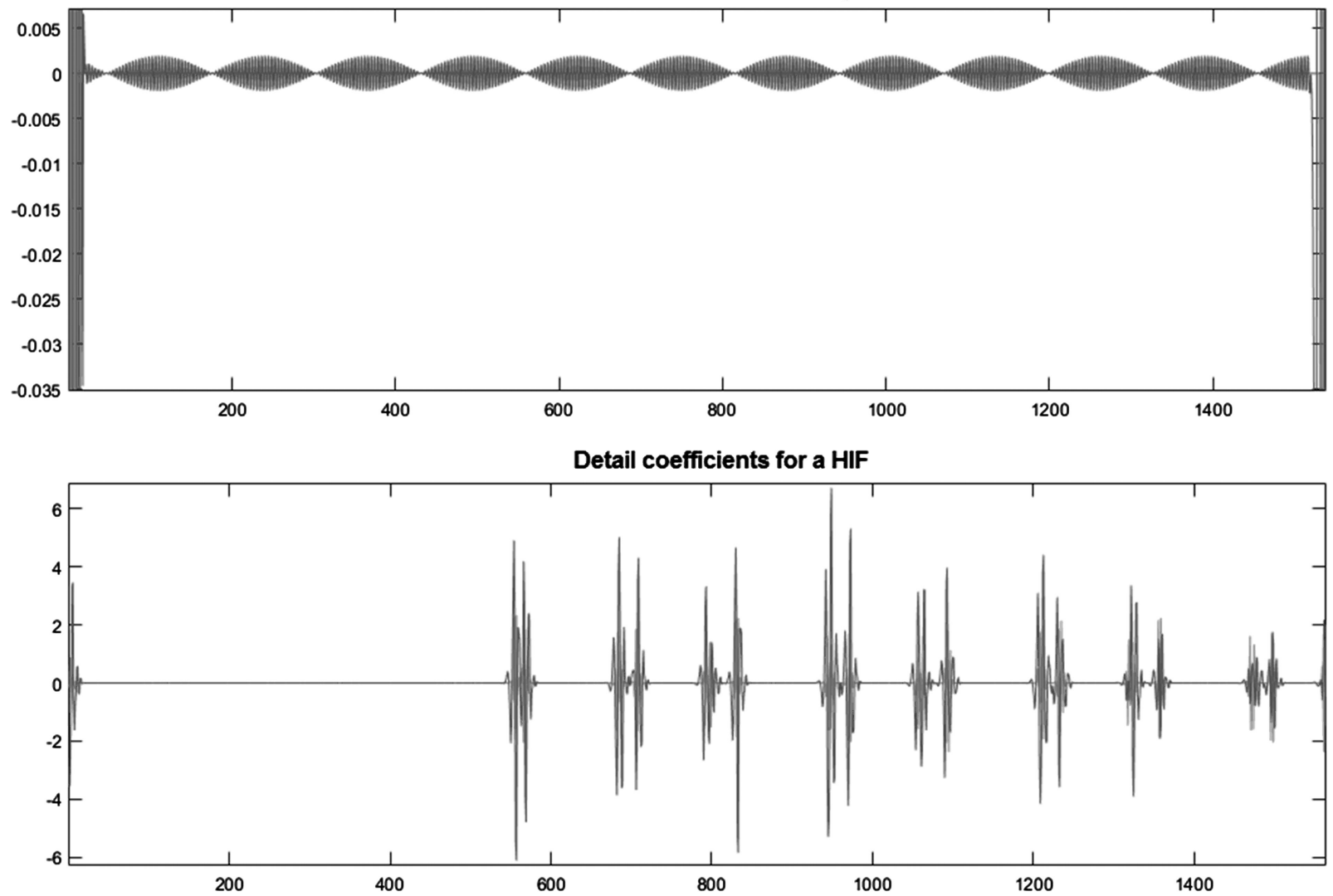

Fig. 3 MRA applied for current oscillographies referred to the system's normal operation and under HIF

systems; for instance, it does not provide the temporal evolution of frequency transients. In this context, the wavelet transform overcomes the limitations of methods based on the Fourier transform.

Multi-resolution analysis (MRA) corresponds to a particular application of the discrete wavelet transform aimed at extracting the signal characteristics. By using a set of filters, the signal is analysed at different resolution levels. At each level, coefficients that describe the signal behaviour at high and low frequencies are obtained [18]. Basically, MRA consists in determining the approximation and detail coefficients. Discrete signal convolution with a low-pass $(h)$ and a high-pass $(g)$ filter generates the approximation and detail coefficients, respectively. The approximation coefficients are described as the high-scale and lowfrequency components of the signal, whereas the detail coefficients are the low-scale and high-frequency components [16]. Fig. 3 shows the application of MRA in current oscillographies referred to the system's normal operation and under HIF.

When applying the MRA, the singularities of the HIF, i.e. the amplitude of high frequency transients are emphasised. Therefore, it is possible to distinguish the normal operation from an HIF, making the diagnosis process more simple, reliable and efficient.

The application of MRA in decomposing a signal generates a considerable number of wavelet coefficients. However, to minimise 


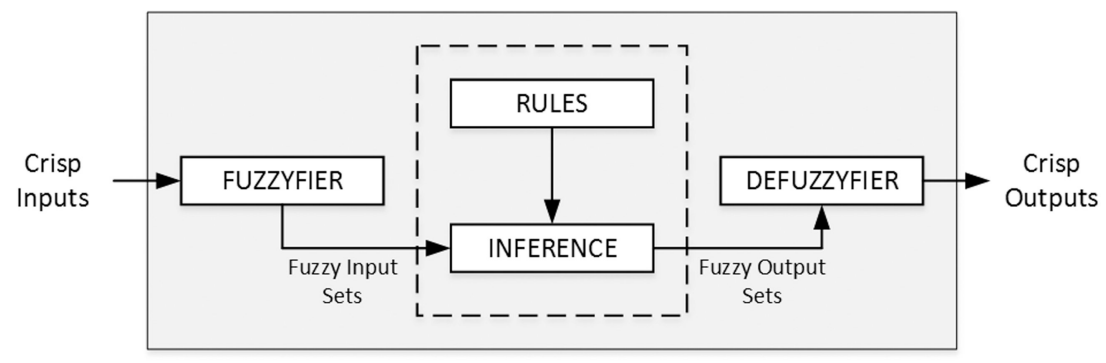

Fig. 4 Fuzzy inference system components

the number of coefficients and improve the methodology efficiency, the energy concept is applied to aggregate the information present in these coefficients [18]. The energy concept is defined by the following equation [15]:

$$
E=\sum_{n=1}^{Z}|x[n]|^{2}
$$

\subsection{Fuzzy inference system}

The FIS performs the mapping of a crisp input vector in a crisp output, i.e. it maps numbers in numbers. It is based on the human capacity in taking decisions in situations where uncertainties and imprecision overcome [19]. The FIS is composed by four components: fuzzyfication, base rules, inference, and defuzzyfication, as shown in Fig. 4.

The presence of the fuzzyfier and defuzzyfier allows the user to work with input and output variables that have real values. All the processes are realised with linguistic variables. In [19-21] can be found a brief explanation about each step of the diagram presented in Fig. 4.

\subsection{Fuzzy-ARTMAP neural network}

The FANN consists of a supervised learning system composed by a pair of adaptive resonance theory [22] modules, fuzzy-ART (responsible for the FANN processing input (vector $\boldsymbol{a}$ )) and fuzzy$\mathrm{ART}_{\mathrm{b}}$ (responsible for the FANN processing output (vector $\boldsymbol{b}$ )), connected by an associative memory module called inter-ART, which is responsible for verifying if there is a match between the FANN input $(\boldsymbol{a})$ and output $(\boldsymbol{b})$. The weight matrices associated with the fuzzy-ART $\mathrm{A}_{\mathrm{a}}$ modules $\left(\boldsymbol{W}^{a}\right)$ and fuzzy-ART $\left(\boldsymbol{W}^{b}\right)$, as well as the inter-ART $\left(\boldsymbol{W}^{a b}\right)$ module, are initialised with values equal to 1 , i.e. every activity is inactive. These activities are activated while occurring the resonance with the input and output patterns. The FANN system presents characteristics of stability, i.e. capacity of learning by adjusting the weights, and plasticity, i.e. capacity of continuing learning when including new patterns without losing the previously acquired knowledge. Therefore, it is possible to enable the continuous training module, allowing the neural system to include new patterns to its memory without needing to reinitialise the training process [23].

Appendix presents a brief description of FANN architecture. Meanwhile, the more complete FANN theory is presented in [22]; the algorithm can be found in [18], and the neural network training flowchart is shown in [24].

\subsection{Evidence theory}

Dempster-Shafer evidence theory [25] is based on probabilistic reasoning and evidence combinations. It is called evidence theory because it deals with the evidence portion and its respective supporting numerical values, associating the relevant portion of a given hypothesis to determine its truth. The computational methodology was developed by Jeffrey Barnett and is presented as follows [26].

This concept considers pieces of evidence in favour and against a given hypothesis to define the accumulated evidence in favour and against the analysed hypothesis. Then, it is possible to define the confidence interval, which is given as

$$
\left[m(\{i\}), \mathfrak{B}^{*}(\{i\})\right]
$$

The probability value $m(\{i\})$ represents the veracity of hypothesis $i$, and the superior probabilistic quantity $\mathfrak{B}^{*}(\{i\})$ represents the chances of failure when one doubts the veracity of hypothesis $i$. The higher the value of $\mathfrak{B}^{*}(\{i\})$, the greater will be the reliability of the tested hypothesis (true or false), which is represented by the value of $m(\{i\})$. Therefore, for any hypothesis tested wills high values for $\mathfrak{B}^{*}(\{i\})$. These values are obtained by using the equations presented in $[18,27,28]$.

\subsection{Voting scheme}

The voting scheme is a simple method that combines the output of a given procedure. A decision is made based on which operating state receives the higher number of votes. The confidence of the decision is represented by the agreement level defined as the ratio between the total number of votes received and the total number of votes $[27,29]$.

\subsection{Detection and classification}

The three-phase current signals of multiple meters allocated along the feeder are decomposed in three levels by multi-resolution analysis, in which the signal windowing and analysis step are defined in one cycle. Due to the great number of coefficients produced in multi-resolution analysis, the energy concept is applied to the third-level detail coefficients, reducing the set to a few scalars. For each measurement bus, the following vector is composed:

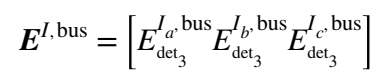

where $E_{\operatorname{det}_{3}}^{I_{a} \text { bus }}, E_{\operatorname{det}_{3}}^{I_{b} \text {, bus }}$, and $E_{\operatorname{det}_{3}}^{I_{c} \text {, bus }}$ denote the energy of the third-level detail coefficients obtained from the current signals (phases $a, b$, and $c$ ) acquired at the measurement point bus; i.e. buses 149, 13, $18,47,54,60,67,76$, and 97.

2.6.1 Methodology based on FIS: On each bus where the signals are acquired there is an FIS responsible for the identification and qualification of the feeder operating state. The input of each FIS (FIS $\left.\boldsymbol{S}_{\text {input }}^{\text {bus }}\right)$ is formed by the vector obtained in (3) from the respective measurement point bus:

$$
\boldsymbol{F I} \boldsymbol{S}_{\text {input }}^{\text {bus }}=\left[\boldsymbol{E}^{I, \text { bus }}\right]
$$

As an example, the FIS in bus 18 has the vector $\boldsymbol{F} I \boldsymbol{S}_{\text {input }}^{18}=\left[\boldsymbol{E}^{I, 18}\right]$ as input; i.e. it uses only the information about the current of bus 18.

After the calculus and evaluations by fuzzy rules, the FIS provides as an output a scalar. To determine the operating state, the membership obtained at the output must be verified by each of the fuzzy sets of its respective FIS. Thus, the obtained result presents a combination of four membership values, i.e. normal operation, HIF at phase $a$, HIF at phase $b$, and HIF at phase $c$, which can be used separately or together. 
Table 1 Operating state output codification

\begin{tabular}{lc}
\hline Operation state & Output codification \\
\hline normal operation & {$\left[\begin{array}{lll}0 & 0 & 0\end{array}\right]$} \\
HIF on phase $a$ & {$\left[\begin{array}{lll}1 & 0 & 1\end{array}\right]$} \\
HIF on phase $b$ & {$\left[\begin{array}{lll}1 & 1 & 0\end{array}\right]$} \\
HIF on phase $c$ & {$\left[\begin{array}{lll}1 & 1 & 1\end{array}\right]$} \\
\hline
\end{tabular}

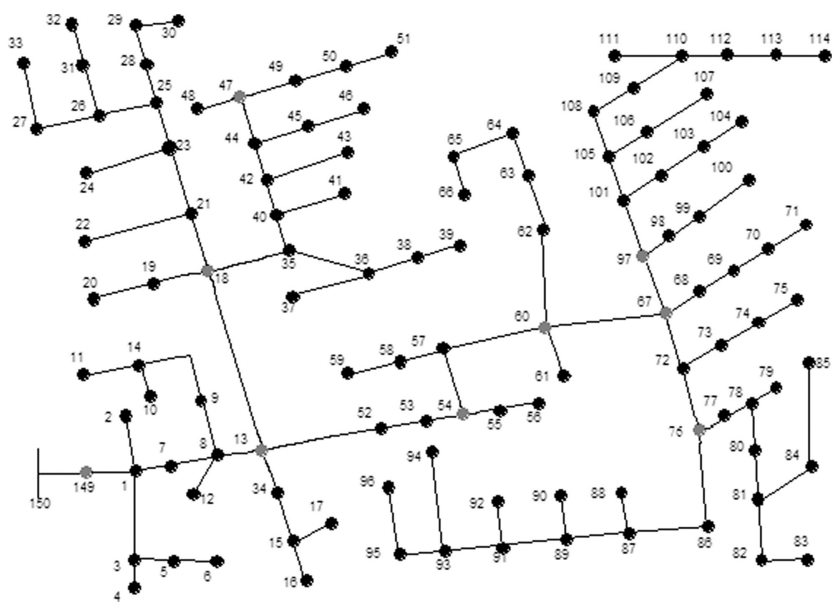

Fig. 5 IEEE-123 distribution feeder

Considering the difficulty presented by the human operators in analysing great quantity of information and, consequently, taking decisions, the evidence theory is used to aggregate the four memberships of the nine FIS. This application simplifies the diagnosis, i.e. the possible operating states contain a probability value and a value indicating its reliability. Therefore, the diagnosis process becomes efficient and reliable, minimising the participation of the human in decision-making event, which provides greater simplicity to the operators' daily routine.

2.6.2 Methodology based on FANN: In this methodology, the vectors obtained in (1) are normalised for use as inputs to multiple neural networks. This normalisation is done by identifying the maximum current value of each analysed vector. This normalisation produces new vectors according to the following equation:

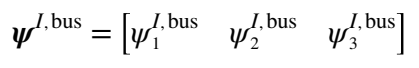

where $\quad \psi_{p}^{I, \text { bus }}=\left(E_{p}^{I, \text { bus }} / E_{\max }^{I, \text { bus }}\right) \quad$ for $\quad p=1,2, \ldots, 3 \quad$ with $E_{\max }^{I, \text { bus }}=\max \left\{E_{p}^{I, \text { bus }}, p=1,2, \ldots, 3\right\}$.

In each bus from which the signals are acquired, there is an FANN, which identifies and qualifies the feeder operating state. The input of each network $\left(\boldsymbol{a}_{\text {input }}\right)$ is formed by the vector $\boldsymbol{\psi}^{I, \text { bus }}$ of the respective measurement point bus

$$
\boldsymbol{a}_{\text {input }}^{\text {bus }}=\left[\psi^{I, \text { bus }}\right]
$$

As an example, the FANN in bus 47 has the vector $\boldsymbol{a}_{\text {input }}^{47}=\left[\boldsymbol{\psi}^{I, 47}\right]$ as input; i.e. it uses only the information about the current of bus 47.

The neural networks provide the system operating state as output $\left(\boldsymbol{b}_{\text {output }}\right)$; e.g. normal operation or operation under an HIF, and the phase in which the failure is present. To represent each operating state, a predefined codification is used according to Table 1.

The output codification is defined as follows: the first bit shows the operating state, and the last two shows the phase under abnormal operation. Since there are two possible operation states, the first bit represents both states; i.e. normal operation ([0]) and HIF ([1]). Similarly, the last two bits show the four combinations of phases under failure; i.e. without the presence of phases ([ $\left.\left[\begin{array}{ll}0 & 0\end{array}\right]\right)$,
Table 2 Training and test sets

\begin{tabular}{lccc}
\hline Failure & \multicolumn{3}{c}{ Pattern set } \\
& Training & Test & Total \\
\hline HIF at phase $a$ & 272 & 66 & 342 \\
HIF at phase $b$ & 234 & 54 & 288 \\
HIF at phase $c$ & 264 & 73 & 333 \\
total & 770 & 193 & 963 \\
\hline
\end{tabular}

presence of phase $a\left(\left[\begin{array}{ll}0 & 1\end{array}\right]\right)$, presence of phase $b\left(\left[\begin{array}{ll}1 & 0\end{array}\right]\right)$, and the presence of phase $c\left(\left[\begin{array}{ll}1 & 1\end{array}\right]\right)$.

Lastly, a simple voting system is used to aggregate the information coming from the intelligent systems presented at the supervised busses, where the final diagnosis will be that predominant at the neural networks output.

\section{Test system and simulations}

Usually, the research centres and utilities do not have a representative database of the distribution system operating under failures. Due to the lack of these information, it is necessary to use a test system to simulate failures, allowing, then, the extraction of pertinent information and the validation of diagnosis and prognosis methodologies.

\subsection{Test system}

This paper is employed the IEEE-123 distribution feeder [30] as test system, which is strongly characterised by the unbalance between phases. Fig. 5 shows the IEEE-123 distribution feeder, modelled with EMTP-RV software.

The current signals acquisition in the distribution feeder above is performed only in the main substation as in other distribution systems. Considering it is a normal practice, one characteristic of the smart grids is the presence of innumerous meters along the feeder. The busses where intelligent meters were allocated are shown in grey in Fig. 5. They were defined after eliminating the single and two-phase sections, remaining only the three-phase sections, and inserting the meters into busses with two or more branches. Therewith, it is possible to evaluate if the bus, where the measurement is realised, interferes on the final result obtained by applying the proposed methodology.

\subsection{Simulations}

A total of 963 HIFs simulations were performed, with a sample frequency of $15.36 \mathrm{kHz}$ (256 samples per cycle), which is commonly used for the measurement/acquisition equipment. The following variables were considered: the failure insertion angle $\left(0^{\circ}\right.$, $45^{\circ}$, and $\left.90^{\circ}\right)$, failure incidence phase $(a, b$, and $c)$, failure bus, and failure behaviour.

Since there is no precise model to represent HIFs, many publications in the literature have proposed models for use in the analysis of such failure [7, 8, 31]. This study used the classical HIF model proposed by Emanuel and Gulachenski [31], which has two direct voltage sources, $V_{p}$ and $V_{n}$, connected in an antiparallel manner by two diodes, as well as a series impedance that controls the arc current magnitude. The HIF model parameters were varied in the following manner: $V_{p}$ from 500 up to $2000 \mathrm{~V} ; V_{n}$ from 2000 up to $2500 \mathrm{~V}$; series resistance from 20 up to $100 \Omega$; and series inductive reactance from 150 up to $300 \Omega$. This variation is necessary to obtain different behaviours of HIFs and its impact in the electric power distribution system.

Neural networks are essential in the proposed methodology. However, if the weights are not adjusted correctly, such as in a previous training, the results produced by the neural networks may not be satisfactory. Then, the selection of patterns to train all neural networks becomes necessary. The simulation pattern set was pseudorandom divided into two: a training set and a test set, as shown in Table 2.

The pattern set used for training the neural networks was also used for validating each FIS, i.e. fuzzy sets and rules. 
Table 3 Fuzzy sets and rules for each measurement point

\begin{tabular}{|c|c|c|c|c|c|c|}
\hline \multirow[t]{2}{*}{ Measurement point } & \multicolumn{3}{|c|}{ Fuzzy sets quantity } & \multicolumn{3}{|c|}{ Quantity of rules } \\
\hline & Input 1 & Input 2 & Input 3 & HIF phase a & HIF phase $b$ & HIF phase $c$ \\
\hline bus 149 & 20 & 14 & 20 & 63 & 45 & 61 \\
\hline bus 13 & 18 & 11 & 15 & 53 & 44 & 60 \\
\hline bus 18 & 16 & 9 & 11 & 55 & 37 & 45 \\
\hline bus 47 & 12 & 14 & 20 & 59 & 46 & 55 \\
\hline bus 54 & 19 & 22 & 21 & 61 & 54 & 74 \\
\hline bus 60 & 20 & 20 & 22 & 57 & 57 & 76 \\
\hline bus 67 & 20 & 17 & 19 & 63 & 52 & 62 \\
\hline bus 76 & 16 & 17 & 16 & 60 & 59 & 65 \\
\hline bus 97 & 18 & 14 & 19 & 65 & 54 & 65 \\
\hline
\end{tabular}

Table 4 FANN parameters for each measurement point

\begin{tabular}{lcccccc}
\hline Measurement point & \multicolumn{3}{c}{ Parameters } \\
& $\alpha$ & & $\beta$ & $\rho_{a}$ & $\rho_{b}$ & $\rho_{a b}$ \\
\hline bus 149 & 0.01 & 1.00 & 0.20 & 1.00 & 0.95 & 0.000001 \\
bus 13 & 0.01 & 1.00 & 0.20 & 1.00 & 0.95 & 0.00001 \\
bus 18 & 0.01 & 1.00 & 0.20 & 1.00 & 0.95 & 0.000001 \\
bus 47 & 0.01 & 1.00 & 0.20 & 1.00 & 0.95 & 0.000001 \\
bus 54 & 0.01 & 1.00 & 0.20 & 1.00 & 0.95 & 0.0001 \\
bus 60 & 0.01 & 1.00 & 0.20 & 1.00 & 0.95 & 0.000001 \\
bus 67 & 0.01 & 1.00 & 0.20 & 1.00 & 0.95 & 0.00001 \\
bus 76 & 0.01 & 1.00 & 0.20 & 1.00 & 0.95 & 0.00001 \\
bus 97 & 0.01 & 1.00 & 0.20 & 1.00 & 0.95 & 0.00001 \\
\hline
\end{tabular}

Table 5 Accuracy rate of the diagnosis module for the two methodologies

\begin{tabular}{lcc}
\hline Measurement bus & \multicolumn{2}{c}{$\begin{array}{c}\text { Accuracy rate, \% } \\
\text { Fuzzy-ARTMAP }\end{array}$} \\
\hline bus 149 & 99.48 & 97.41 \\
bus 13 & 100.00 & 98.96 \\
bus 18 & 99.48 & 97.41 \\
bus 47 & 97.41 & 95.85 \\
bus 54 & 99.48 & 95.85 \\
bus 60 & 99.48 & 98.96 \\
bus 67 & 100.00 & 95.85 \\
bus 76 & 97.93 & 98.96 \\
bus 97 & 100.00 & 100.00 \\
\hline
\end{tabular}

Table 6 Principal characteristics comparison between the methodology employing FIS and the methodology using FANN

\begin{tabular}{|c|c|c|}
\hline \multirow[t]{2}{*}{ Characteristic } & \multicolumn{2}{|c|}{ Intelligent tool } \\
\hline & FIS & Fuzzy-ARTMAP \\
\hline dependency of human operator & high & low \\
\hline Is it necessary a previous training? & no & yes \\
\hline training time & - & $1.30 \mathrm{~s}$ \\
\hline diagnosis time & $27.17 \mathrm{~ms}$ & $36.69 \mathrm{~ms}$ \\
\hline $\begin{array}{l}\text { Does it allow using the evidence theory } \\
\text { at the final? }\end{array}$ & yes & no \\
\hline $\begin{array}{l}\text { Does it allow using new faults at the } \\
\text { future? }\end{array}$ & yes & yes \\
\hline difficulty in including new faults & high & low \\
\hline generalisation capacity & high & high \\
\hline global efficiency & $99.25 \%$ & $97.69 \%$ \\
\hline
\end{tabular}

\section{Conception of the HIF diagnosis modules}

The diagnosis modules' rules and input fuzzy sets of the first methodology, and neural networks parameters of the second methodology were defined based on the 963 HIF simulated cases.
Table 3 presents the fuzzy sets quantity for each input and the quantity of rules, considering the measurement bus.

In the output of each FIS was employed four fuzzy sets; i.e. normal operation, HIF phase $a$, HIF phase $b$, and HIF phase $c$; which are the same for all the measurement points. It should be highlighted that the fuzzy set and rules definition are dependent on the operators' analysis experience.

The values of the neural networks parameters used, considering each measurement bus, are presented in Table 4 . The parameter $\underline{\rho}_{a}$ is named $\rho$ baseline and is adapted using successive increment $\varepsilon$ until it reaches maximum similarity (MS). MS occurs when (14) is satisfied [22].

\section{Results and discussion}

There are two fundamental steps in the development of an efficient failure diagnosis system: signature extraction and the use of modern tools for data analysis.

In the first step of the failure diagnosis process, i.e. the extraction of current singularities, the Daubechies mother wavelet with a fourth-order filter $(d b 4)$ is applied. The use of the wavelet transform allows the identification of frequency transients of HIF. It was only selected the characteristics obtained from three-phase current signals to become the methodology simple and reduce its costs inherent to the real-time implementation, i.e. cheaper data acquisition and processing modules. The use of multiple characteristics, e.g. voltage, is possible and can improve the diagnosis system reliability, however it elevates utilities' initial investments. The behaviour indices extracted from the oscillographies are evaluated by multiple FIS and multiple FANNs. Table 5 presents the accuracy rate of the two methodologies developed for the detection and classification of HIF in electric power distribution systems.

Regarding the normal system operation, it is emphasised that the two methodologies presented $100 \%$ accuracy in diagnosis.

The principal objective of this paper is to develop efficient systems to aid the operators in HIF diagnosis process and carefully analyse the relevant characteristics for the automation process. Table 6 presents an evaluation of the essential attributes for realtime applications in modern electrical distribution systems. After, these characteristics are carefully discussed. 
Table 7 Membership values for the output fuzzy sets

\begin{tabular}{|c|c|c|c|c|c|c|c|c|c|}
\hline Bus & 149 & 13 & 18 & 47 & 54 & 60 & 67 & 76 & 97 \\
\hline$\overline{\mathrm{HIF} a}$ & $100 \%$ & $100 \%$ & $56.37 \%$ & $89.98 \%$ & $78.66 \%$ & $78.63 \%$ & $100 \%$ & $98.27 \%$ & $100 \%$ \\
\hline HIF $b$ & $0 \%$ & $0 \%$ & $43.63 \%$ & $10.02 \%$ & $21.34 \%$ & $21.37 \%$ & $0 \%$ & $1.73 \%$ & $0 \%$ \\
\hline HIF $c$ & $0 \%$ & $0 \%$ & $0 \%$ & $0 \%$ & $0 \%$ & $0 \%$ & $0 \%$ & $0 \%$ & $0 \%$ \\
\hline
\end{tabular}

Table 8 Diagnosis provided by the neural networks

\begin{tabular}{lc}
\hline Neural network & Diagnosis \\
\hline bus 149 & phase $b$ \\
bus 13 & phase $b$ \\
bus 18 & phase $c$ \\
bus 47 & phase $b$ \\
bus 54 & phase $a$ \\
bus 60 & phase $c$ \\
bus 67 & phase $b$ \\
bus 76 & phase $b$ \\
bus 97 & phase $c$ \\
\hline
\end{tabular}

The human operators' dependency is due to the necessity in analysing oscillographies or characteristics index to determine the operational conditions. This procedure demands time and can generate errors caused by the human operators. In this item, the FIS is more critical due to the great quantity of fuzzy rules, whilst the FANN only needs the values of the parameters $\alpha, \beta, \rho_{a}, \rho_{b}, \rho_{a b}$, and $\varepsilon$

Although is easier to define the topology of a neural network, a previous training is necessary to provide satisfactory final results. However, the neural network function can be compromised if there is not enough fault patterns, causing undesirable errors on diagnosis. The training time of the FANN is around $1.30 \mathrm{~s}$, which can be reduced applying parallel processing technique, e.g. FPGA and DSP.

Analysing the two techniques used, the one that provided the information of the system operating state in less time was the FIS. Nevertheless, the FANN, even with processing time a little higher, is a good option to real-time applications.

When using multiple measurement points, where each one provides the feeder operational condition, the operator might not be capable to aggregate such information and not take an adequate preventive/corrective action as necessary. Thereby, fusion information techniques must be applied to reduce the quantity of diagnosis, and if possible, with a probabilistic value. The evidence theory is an efficient tool with this characteristic. This work uses the evidence theory only for FIS due to the way the results are produced. An example showing how to proceed to apply the evidence theory is presented below.

Firstly, consider the centre areas values generated by the FIS for each bus, being them: 6.25 (bus 149), 6.25 (bus 13), 8.98 (bus 18), 6.88 (bus 47), 7.58 (bus 54), 7.59 (bus 60), 6.25 (bus 67), 6.36 (bus 76), and 6.25 (bus 97). Rebutting these values on the output fuzzy sets, obtains the membership values as shown in Table 7 .

Then, the values presented at this table are aggregated by evidence theory, producing the following confidence intervals: HIF $a \quad[99.99 \%$ 99.99\%], HIF $b[0.00 \% 0.00 \%]$, and $\mathrm{HIF} c$ [ $0.00 \% 0.00 \%]$. Thus, the decision-making is based on the intervals evaluation and not anymore dependent of the operators experience.

Although is not possible to apply the evidence theory on the results provided by the neural networks it is possible to generate a confidence value by applying a voting scheme. The theory of evidence is impossible to be applied with the FANN due to the form how the neural system provides the final result, i.e. only one diagnosis with an assurance of $100 \%$, different from the FIS, which provide respective probabilities for each of the three possible diagnoses. Consider as an example an HIF at phase $b$ where the diagnosis results are shown in Table 8.

Observing the results, the diagnoses have no probabilistic value, which not allows to aggregate them. With the use of the scheme, the operator is able to point the fault that is the most probably by analysing a perceptual value of votes received for each one of the possible states. The final result of the example is (considering Table 8): phase $a-11.11 \%$, phase $b-55.56 \%$, and phase $c-$ $33.33 \%$.

Regarding the inclusion of new faults, both the techniques allow this procedure to be done. The difference is in the way that the inclusion is performed, once the neural network activates the continuous training module, the FIS needs new fuzzy sets. The new fuzzy sets inclusion demands time by the operators and can generate rough errors. Thus, the continuous training module consists in a robust and reliable tool compared to the FIS way of including new faults.

Lastly, evaluating the global efficiency of each tool, is concluded that both presents high generalisation capacity, once they can distinguish faults in different phases $(a, b$, and $c)$, with different incidence angles $\left(0^{\circ}, 45^{\circ}\right.$, and $\left.90^{\circ}\right)$, at distinct points of the distribution feeder

\section{Conclusions}

This paper compares two fuzzy based methodologies for failure diagnosis in electric power distribution systems. The method was developed to detect and classify HIFs, which represent a huge problem for utilities due to the possibility of evolving into a critical event that could cause energy supply interruption. Through the application of the developed methods, it is possible to avoid major prejudices to utilities and consumers.

For abnormality detection on the feeder, it is necessary to extract the singularities present in current signals, allowing the representation of the possible operating states of the feeders. As stated in the smart grid paradigm, the method uses oscillographies from different points along the feeder, providing complete and comprehensive information in relation to the buses and their operating states. Analysis of the diagnosis and prognosis system is automatically carried out by using the wavelet transform, artificial neural networks, and evidence theory. Through the combined use of signal processing techniques, artificial intelligence algorithms, and data fusion techniques, high generalisation capacity, flexibility, robustness, and efficiency are obtained, which are important characteristics that aid the decision-making process. First, to detect and classify abnormalities, current oscillographies are evaluated in the wavelet domain search of frequency transients. Nine FIS and nine FANNs provide the system operating state, with the Dempster-Shafer evidence theory and voting scheme generating the final state, respectively.

To evaluate the performance of the proposed methodologies, HIFs were simulated in a distribution feeder with 123 buses. The results show that the algorithm is efficient, reliable, and robust, mainly when applied to the diagnosis of HIFs; this represents an advance in this context considering that such faults have complex behaviours and characteristics.

\section{Acknowledgments}

The authors thank the São Paulo Research Foundation (FAPESP) for their financial support for this research.

\section{References}

[1] Das, R., Madani, V., Aminifar, F., et al.: 'Distribution automation strategies: evolution of technologies and the business case', IEEE Trans. Smart Grid, 2015, 6, (4), pp. 2166-2175

[2] Madani, V., Das, R., Aminifar, F., et al.: 'Distribution automation strategies challenges and opportunities in a changing landscape', IEEE Trans. Smart Grid, 2015, 6, (4), pp. 2157-2165 
[3] Kim, M., Metzner, J.J.: 'A key exchange method for intelligent electronic devices in distribution automation', IEEE Trans. Power Deliv., 2010, 25, (3), pp. 1458-1464

[4] Lo, C.H., Ansari, N.: 'Decentralized controls and communications for autonomous distribution networks in smart grid', IEEE Trans. Smart Grid, 2013, 4, (1), pp. 66-77

[5] Macedo, M.N.Q., Galo, J.J.M., Almeida, L.A.L., et al:: 'Methodology for the calculation of the factor of priority for smart grid implantation using fuzzy logic', Int. J. Electr. Power Energy Syst., 2016, 78, (6), pp. 563-568

[6] Tcheou, M.P., Lovisolo, L., Ribeiro, M.V., et al.: 'The compression of electric signal waveforms for smart grids: state of the art and future trends', IEEE Trans. Smart Grid, 2014, 5, (1), pp. 291-302

[7] Samantaray, S.R., Dash, P.K., Upadhyay, S.K.: 'Adaptive Kalman filter and neural network based high impedance fault detection in power distribution networks', Int. J. Electr. Power Energy Syst., 2009, 31, (4), pp. 167-172

[8] Samantaray, S.R.: 'Ensemble decision trees for high impedance fault detection in power distribution network', Int. J. Electr. Power Energy Syst., 2012, 43, (1), pp. 1048-1055

[9] Gautam, S., Brahma, S.M.: 'Detection of high impedance fault in power distribution systems using mathematical morphology', IEEE Trans. Power Syst., 2013, 28, (2), pp. 1226-1234

[10] Torres, V., Guardado, J.L., Ruiz, H. et al.: 'Modeling and detection of high impedance faults', Int. J. Electr. Power Energy Syst., 2014, 61, (10), pp. 163172

[11] Ghaderi, A., Mohammadpour, H.A., Ginn, H.L., et al.: 'High-impedance fault detection in the distribution network using the time-frequency-based algorithm', IEEE Trans. Power Deliv., 2015, 30, (3), pp. 1260-1268

[12] Macedo, J.R., Resende, J.W., Bissochi, C.A., et al.: 'Proposition of an interharmonic-based methodology for high-impedance fault detection in distribution systems', IET Gener. Transm. Distrib., 2015, 9, (16), pp. 25932601

[13] Batista, O.E., Flauzino, R.A., Araujo, M.A., et al.: 'Methodology for information extraction from oscillograms and its application for highimpedance faults analysis', Int. J. Electr. Power Energy Syst., 2016, 76, (3), pp. 23-34

[14] Costa, F.B. Souza, B.A., Brito, N.S.D et al: 'Real-time detection of transients induced by high-impedance faults based on the boundary wavelet transform', IEEE Trans. Ind. Appl., 2015, 51, (6), pp. 5312-5323

[15] Oppenheim, A.V., Schafer, R.W.: 'Digital signal processing' (Prentice-Hall, 1975, US edn.)

[16] Mallat, S.: 'A wavelet tour of signal processing: the sparse way' (Academic Press, 2009, 3rd edn.)

[17] Daubechies, I.: 'Ten lectures on wavelets' (Society for Industrial and Applied Mathematics, 1992, 1st edn.)

[18] Decanini, J.G.M.S., Tonelli-Neto, M.S., Minussi, C.R. 'Robust fault diagnosis in power distribution systems based on fuzzy ARTMAP neural network-aided evidence theory', IET Gener. Transm. Distrib., 2012, 6, (11), pp. $1112-1120$

[19] Mendel, J.M.: 'Fuzzy logic systems for engineering: a tutorial', Proc. IEEE, 1995, 83, (3), pp. 345-377

[20] Kandel, A.: 'Fuzzy control systems' (CRC Press, 1993, 1st edn.)

[21] Chen, W.H., Liu, C.W., Tsai, M.S.: 'On-line fault diagnosis of distribution substations using hybrid cause-effect network and fuzzy rule-based method', IEEE Trans. Power Deliv., 2000, 15, (2), pp. 710-717

[22] Carpenter, G.A., Grossberg, S., Markuzon, N., et al.: 'Fuzzy ARTMAP: a neural network architecture for incremental supervised learning of analog multidimensional maps', IEEE Trans. Neural Netw., 1992, 3, (5), pp. 698-713

[23] Barros, A.C., Tonelli-Neto, M.S., Decanini, J.G.M.S., et al.: 'Detection and classification of voltage disturbances in electrical power systems using a modified Euclidean ARTMAP neural network with continuous training', Electr. Power Compon. Syst., 2015, 43, (19), pp. 1-11

[24] Decanini, J.G.M.S., Tonelli-Neto, M.S., Malange, F.C.V., et al.: 'Detection and classification of voltage disturbances using a fuzzy-ARTMAP-wavelet network', Electr. Power Syst. Res. , 2011, 81, (12), pp. 2057-2065

[25] Shafer, G.: 'A mathematical theory of evidence' (Princeton University Press, 1976, 1st edn.)

[26] Barnett, J.A.: 'Computational methods for a mathematical theory of evidence', Classic Works of the Dempster-Shafer Theory of Belief Functions, 2008, 219, pp. 197-216

[27] Santoso, S., Powers, E.J., Grady, W.M., et al.: 'Power quality disturbance waveform recognition using wavelet-based neural classifier - part 1: theoretical foundation', IEEE Trans. Power Deliv., 2000, 15, (1), pp. 222-228

[28] Buchanan, B.G., Shortliffe, E.D.: 'Rule-based expert systems: the MYCIN experiments of the Stanford heuristic programming project' (Addison-Wesley, 1984)

[29] Battiti, R., Colla, A.M.: 'Democracy in neural nets: voting schemes for classification', Neural Netw., 1994, 7, (4), pp. 691-707

[30] IEEE Power Engineering Society: 'IEEE 123 node test feeder', Distribution System Analysis Subcommittee, 2013. Available at: http:// ewh.ieee.org/soc/pes/dsacom/testfeeders/feeder123.zip

[31] Emanuel, A.E., Gulachenski, E.M.: 'High impedance fault arcing on sandy soil in $15 \mathrm{kV}$ distribution feeders: contributions to the evaluation of the low frequency spectrum', IEEE Trans. Power Deliv., 1990, 5, (2), pp. 676-686

[32] Zadeh, L.A.: 'Fuzzy sets', Inf. Control, 1965, 8, pp. 338-353

\section{Appendix}

\subsection{FANN algorithm}

The FANN architecture (Fig. 6) consists, basically, of the following procedures [22]:

I. Fuzzy-ART $\mathrm{a}$ and fuzzy-ART $\mathrm{A}_{\mathrm{b}}$ modules: The input data are allocated in the vector $\boldsymbol{a}=\left[\begin{array}{lll}a_{1} & \ldots & a_{M_{a}}\end{array}\right]$, i.e. vector $\boldsymbol{a}$ has $M_{a}$ components, and the output data compose the vector $\boldsymbol{b}=\left[\begin{array}{lll}b_{1} & \ldots & b_{M_{b}}\end{array}\right]$, i.e. vector $\boldsymbol{b}$ has $M_{b}$ components. These vectors are normalised in order to meet the criterion of fuzzy logic [32], belonging to the interval $[0,1]$. The input and output vectors are denoted by the vectors $\boldsymbol{I}^{a}$ and $\boldsymbol{I}^{b}$, respectively,

$$
\begin{aligned}
\boldsymbol{I}^{a} & =\left[\begin{array}{ll}
\boldsymbol{a} & \boldsymbol{a}^{c}
\end{array}\right]=\left[\begin{array}{llllllll}
a_{1} & a_{2} & \ldots & a_{M a} & a_{1}^{c} & a_{2}^{c} & \ldots & a_{M a}^{c}
\end{array}\right] \\
\boldsymbol{I}^{b} & =\left[\begin{array}{ll}
\boldsymbol{b} & \boldsymbol{b}^{c}
\end{array}\right]=\left[\begin{array}{llllllll}
b_{1} & b_{2} & \ldots & b_{M b} & b_{1}^{c} & b_{2}^{c} & \ldots & b_{M b}^{c}
\end{array}\right]
\end{aligned}
$$

The encoding of the complement is performed to preserve the amplitude information: $a_{i}^{c}=1-a_{i}$ and $b_{i}^{c}=1-b_{i}$.

II. Weights matrices: Initially, all weights of the fuzzy-ART ${ }_{\mathrm{a}}$, fuzzy-ART $\mathrm{b}$ and inter-ART matrices $\left(\boldsymbol{W}_{\left(N x_{2} M a\right)}^{a}, \boldsymbol{W}_{(N x 2 M b)}^{b}\right.$, and $\left.\boldsymbol{W}_{(N x N)}^{a b}\right)$ have a value equal to one, indicating that there is no category active, where $N$ is the number of training patterns.

III. Neural network parameters: Parameters used in the processing of the FANN are:

- Choice parameter $\alpha(\alpha>0)$ : selects the categories.

- Training rate $\beta(\beta \in[0,1])$ : controls the network adaptation velocity.

- Vigilance parameter $\rho(\rho \in[0,1])$ : responsible for the number of categories created, i.e. the resonance of the network.

- Increment parameter $\varepsilon$ (positive and small): an internal mechanism, denominated match-tracking, is responsible for the network auto-regulator process, which maximises the generalisation and minimises the error. If the network prognosis is wrong, through an instructed associative connection, the fuzzy-ART $\mathrm{A}_{\mathrm{a}}$ vigilance parameter is incremented by a minimal value $(\varepsilon)$ to correct the fuzzy-ART $\mathrm{b}_{\mathrm{b}}$ error.

IV. Choice of categories in fuzzy-ART modules: The fuzzy-ART choice function $\left(T_{j}^{a}\right)$ is calculated:

$$
T_{j}^{a}=\frac{\left|\boldsymbol{I}^{a} \wedge \boldsymbol{W}_{j}^{a}\right|}{\alpha+\left|\boldsymbol{W}_{j}^{a}\right|}
$$

where $\wedge$ is the AND fuzzy operator, i.e. $\boldsymbol{I} \wedge \boldsymbol{W} \equiv \min \left(I_{i}, W_{i}\right)$. Choose the winner category of fuzzy-ART $\mathrm{A}_{\mathrm{a}}$ :

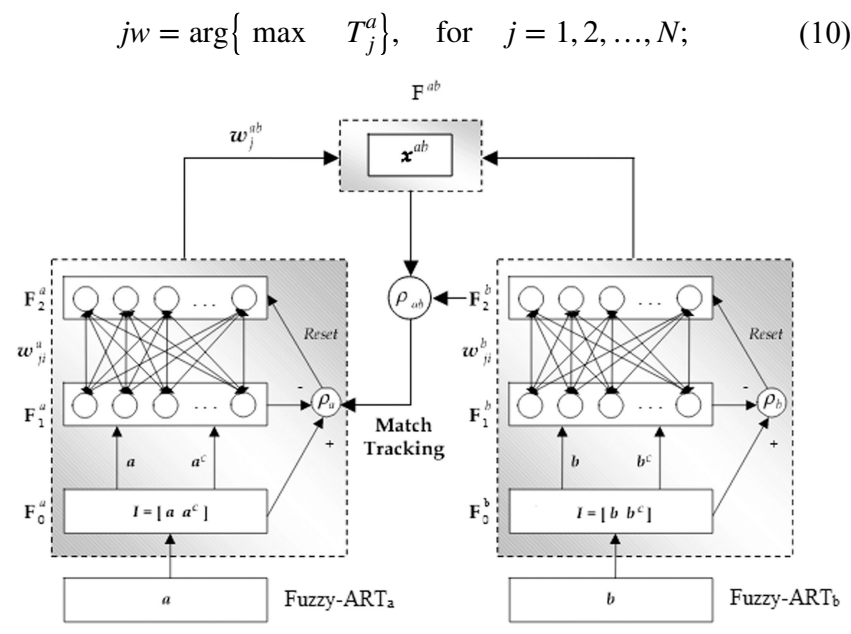

Fig. 6 FANN architecture 
The same procedure applies to the Fuzzy- $\mathrm{ART}_{\mathrm{b}}$ module, using the index $k$, instead of the index $j$, to identify the Fuzzy-ART $\mathrm{b}_{\mathrm{b}}$ module. V. Activity vector: The activity vector on F2 layer is defined by $\boldsymbol{Y}^{b}=\left[\begin{array}{lll}Y_{1}^{b} & \ldots & Y_{N}^{b}\end{array}\right]$, where $Y_{k w}^{b}=1$ and $Y_{k}^{b}=0$ for $k=1,2, \ldots, N$; $k \neq k w$.

VI. Vigilance criterions: The verification of vigilance criterions of the fuzzy-ART $\mathrm{a}_{\mathrm{a}}, \mathrm{Fuzzy}_{\mathrm{ART}}$, and inter-ART modules occurs through (11)-(13), respectively,

$$
\frac{\left|\boldsymbol{I}^{a} \wedge \boldsymbol{W}_{j w}^{a}\right|}{\left|\boldsymbol{I}^{a}\right|} \geq \rho_{a}
$$

If not, perform the reset, i.e. attribute $T_{j w}^{a}=0$ and choose again the winner category of fuzzy-ART ${ }_{\mathrm{a}}$

$$
\frac{\left|\boldsymbol{I}^{b} \wedge \boldsymbol{W}_{k w}^{b}\right|}{\left|\boldsymbol{I}^{b}\right|} \geq \rho_{b}
$$

If not, perform the reset, i.e. attribute $T_{k w}^{b}=0$ and choose again the winner category of fuzzy-ART

$$
\frac{\left|\boldsymbol{Y}^{b} \wedge \boldsymbol{W}_{j w}^{a b}\right|}{\left|\boldsymbol{Y}^{b}\right|} \geq \rho_{a b}
$$

If not, increment the vigilance parameter $\rho_{a}$, $\rho_{a}=\left(\left|\boldsymbol{I}^{a} \wedge \boldsymbol{W}_{j w}^{a}\right| /\left|\boldsymbol{I}^{a}\right|\right)+\varepsilon$, attribute $T_{j w}^{a}=0$ and choose again the winner category of fuzzy-ART . $_{\text {. }}$

VII. Weights adaptation: The weights adaptation of the fuzzy-ART and fuzzy-ART $\mathrm{A}_{\mathrm{b}}$ modules is performed using (14) and (15), respectively,

$$
\begin{aligned}
& \boldsymbol{W}_{j w}^{a(\text { new })}=\beta\left(\boldsymbol{I}^{a} \wedge \boldsymbol{W}_{j w}^{a \text { (old) }}\right)+(1-\beta) \boldsymbol{W}_{j w}^{a(\text { old })} \\
& \boldsymbol{W}_{k w}^{b(\text { new })}=\beta\left(\boldsymbol{I}^{b} \wedge \boldsymbol{W}_{k w}^{b \text { (old) }}\right)+(1-\beta) \boldsymbol{W}_{k w}^{b(\text { old })}
\end{aligned}
$$

Finally, the weights of inter-ART module are adapted

$$
\boldsymbol{W}_{j w}^{a b}=\left[\begin{array}{llll}
W_{j w, 1}^{a b} & W_{j w, 2}^{a b} & \cdots & W_{j w, N}^{a b}
\end{array}\right]
$$

where $W_{j w, k w}^{a b}=1$ and $W_{j w, k}^{a b}=0$, for $k=1,2, \ldots, N ; k \neq k w$. 